\title{
A Within-host Tuberculosis Model Using Optimal Control
}

\author{
Yudi Ari Adi \\ Department of Mathematics, Ahmad Dahlan University, Yogyakarta, Indonesia \\ yudi.adi@math.uad.ac.id
}

\begin{abstract}
Article History:

Received : 05-01-2021

Revised : 17-03-2021

Accepted : 20-03-2021

Online : :16-04-2021

\section{Keywords:}

Tuberculosis; Mathematical model; Optimal control;

In this paper, we studied a mathematical model of tuberculosis with vaccination for the treatment of tuberculosis. We considered an in-host tuberculosis model that described the interaction between Macrophages and Mycobacterium tuberculosis and investigated the effect of vaccination treatments on uninfected macrophages. Optimal control is applied to show the optimal vaccination and effective strategies to control the disease. The optimal control formula is obtained using the Hamiltonian function and Pontryagin's maximum principle. Finally, we perform numerical simulations to support the analytical results. The results suggest that control or vaccination is required if the maximal transmission of infection rate at which macrophages became infected is large.
\end{abstract} Hamiltonian and Pontryagin function.

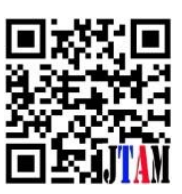

\section{A. INTRODUCTION}

Tuberculosis (TB) is the world's leading infectious disease caused by Mycobacterium tuberculosis (MTb). Many attempts have been proposed to explain the progression of TB disease by considering the influencing factors, including the mechanisms of bacterial response and immunity. However, the factors that influence the development of TB are not currently known with certainty. Motivated by this, we consider a within-host tuberculosis model in (Adi \& Thobirin, 2020) with three components: uninfected macrophages, infected macrophages $\left(M_{i}\right)$, and MTb bacteria, denoted by $M_{u}, M_{i}$, and $B$, respectively. The model is given in the following form of ordinary differential equation system

$$
\begin{aligned}
\frac{d M_{u}}{d t} & =\Lambda-\mu M_{u}-\frac{\beta M_{u} B}{1+\alpha B}, \\
\frac{M_{i}}{d t} & =\frac{\beta M_{u} B}{1+\alpha B}-c M_{i}-\frac{k M_{i}}{1+\varepsilon M_{i}}, \\
\frac{d B}{d t} & =r c M_{i}-\gamma M_{u} B-d B .
\end{aligned}
$$


In this model (1.1), $\Lambda$ and $\mu$ are a constant production rate and a natural death rate of uninfected macrophages, respectively. Parameter $\beta$ is the maximal transmission of infection rate at which macrophages became infected at a saturated incidence rate of $\frac{\beta M_{u} B}{1+\alpha B}$, whit $\frac{1}{1+\alpha B}$ is an inhibition effect. Parameter $r$ and $c$ are the average numbers of the MTb bacteria released by infected macrophages and the rate of macrophages burst, respectively. The parameters $\gamma$ is the MTb bacteria death rate by uninfected macrophages, and $d$ is MTb bacteria natural death rate. The infected macrophages die due to an adaptive immune response modeled in a density-dependent term, $\frac{k M_{i}}{1+\varepsilon M_{i}}$, whereas $k$ is the maximum killing rate, and $\varepsilon$ is a halfsaturation constant.

In many works of literature of tuberculosis models, it is usually considered drug administration or vaccination, as in (Baba et al., 2020; Blaser et al., 2016; Brooks-Pollock et al., 2014; Byrne et al., 2015; Kim et al., 2018; Kuddus et al., 2020; Kyu et al., 2018). Many of the TB models use optimal control theory regarding their treatment strategies (Agusto \& Adekunle, 2014; Baba et al., 2020; Bowong, 2010; Choi et al., 2015; Emvudu et al., 2011; Fatmawati et al., 2020; Gao \& Huang, 2018; Moualeu et al., 2015). In a within-host tuberculosis model, the immune response to bacteria is generally assumed, as in (Adi \& Thobirin, 2020; Zhang, 2020; Zhang et al., 2020). This paper will apply the optimal control theory to a within-host tuberculosis model by considering the vaccination that affects macrophages.

The purpose of this study is to determine the treatment strategies in a within-host tuberculosis model. We apply optimal control theory to determine optimal treatment that will maximize tuberculosis prevention efforts, such as vaccinations.

\section{B. METHODS}

In this section, in order to obtain the optimal strategies, we recall the basic theory of optimal control (Chambers et al., 1965). We consider a system of ordinary differential equations

$$
\dot{\mathbf{x}}=\mathbf{f}(\mathbf{x}(t)), \mathbf{x}(0)=\mathbf{x}_{0},
$$

where $\mathbf{x}_{0} \in \mathbb{R}, \mathbf{f}: \mathbb{R}^{n} \rightarrow \mathbb{R}^{n}$, and $\mathbf{x}:[0, \infty) \rightarrow \mathbb{R}^{n}$. Suppose that the right-hand side depends on a parameter $u:[0, \infty) \rightarrow A$, where $A \subset \mathbb{R}^{m}$. Then the system becomes

$$
\dot{\mathbf{x}}=\mathbf{f}(\mathbf{x}(t), u(t)), \mathbf{x}(0)=\mathbf{x}_{0}, \mathbf{x}(T) \text { free } .
$$

In this system, the solution $\mathbf{x}(t)$ depends on the control $u(t)$. The corresponding response of the system is a trajectory that corresponds to the control $u(t)$. In system (2.2), the control may be arbitrary, so the problem does not have a solution. Therefore, we need to find the best control to minimize or maximize the performance measure as an objective function. In a disease-control model, we need to find the control to minimize the cost of controlling the disease. For that purpose, a payoff functional is defined as follows

$$
J[u]=\int_{0}^{T} g(\mathbf{x}(t), u(t)) d t,
$$

where $\mathbf{x}(t)$ solves (2.2) for the specified control $u(t)$, with the given function $g: \mathbb{R}^{n} \times A \rightarrow$ $\mathbb{R}$ and terminal time $T$ as well. The function $g$ is called the running payoff. Now, introduce the admissible controls

$$
\Omega=\left\{u(t) \in L^{1}(0, T): u(t) \in A\right\} .
$$

The optimal control problem is to find a control $u^{*}(t) \in \Omega$ that minimizes or maximizes the payoff functional (2.3). A corresponding solution together with the optimal control gives the pair of optimal control $\left(\mathbf{x}^{*}, u^{*}\right)$.

If such a control $u(t)$ exists, it is called the optimal control. For maximum problem, the solution $\left(\mathbf{x}^{*}, u^{*}\right)$, if exits, can be found by Pontryagin's maximum principle. According to the constraint in the Lagrangian problem, the time-varying Lagrange multiplier $\lambda(t)$ was 
introduced. The function $\lambda(t)$ is usually called an adjoint variable of the system. The comparable function, in this case, is the Hamiltonian function $H$, defined for all $t \in[0, T]$ by

$$
H(\mathbf{x}(t), u(t), \lambda(t))=g(\mathbf{x}(t), u(t))+\sum_{i=1}^{n} \lambda_{i}(t) f_{i}(\mathbf{x}(t), u(t)) .
$$

The Pontryagin maximum principle is precisely formulated as follows .

Theorem 1. Let $u^{*}(t)$ be a piecewise control defined on $[0, T]$ and $\boldsymbol{x}^{*}(t)$ be the associated trajectory. Then there exists a nonzero adjoint vector function $\lambda^{*}(t)$ that is a solution to the adjoint system

$$
\begin{aligned}
& \dot{\lambda}(t)=-\frac{\partial H(\mathbf{x}(t), u(t), \lambda(t))}{\partial x} \\
& \lambda(T)=0,
\end{aligned}
$$

so that $\mathbf{x}^{*}(t)$ maximizes $H\left(\mathbf{x}^{*}(t), u(t), \lambda(t)\right)$ for $u(t) \in \Omega$, that is

$$
H\left(\mathbf{x}^{*}(t), u^{*}(t), \lambda^{*}(t)\right) \geq H\left(\mathbf{x}^{*}(t), u(t), \lambda^{*}(t)\right), \text { for all } u(t) \in \Omega .
$$

Thus, the necessary conditions for optimizing the Hamiltonian are:

$$
\begin{array}{ll}
\frac{\partial H}{\partial u} & =0 \Rightarrow g_{u}+\sum_{i=1}^{n} \lambda_{i}(t)\left(f_{i}\right)_{u}=0, \\
\dot{\lambda}_{i}(t) & =-\frac{\partial H(\mathbf{x}(t), u(t), \lambda(t))}{\partial x_{i}} \Rightarrow \dot{\lambda}_{i}(t)=-g_{x_{i}}-\sum_{i=1}^{n} \lambda_{i}(t)\left(f_{i}\right)_{x_{i}}, \\
\lambda(T) & =0 .
\end{array}
$$

Please refer to Pontryagin's book (Kaufman, 1964) and some extensions book, such as (Becker et al., 1989; Seierstad \& Sydsaeter, 1977) for more details.

\section{RESULT AND DISCUSSION}

\section{Optimal Control Problem}

This section reformulates and analyzes an optimal control problem for the model (1.1) to determine the optimal trajectories of uninfected macrophages, infected macrophages, and MTb bacteria in response to the optimal strategy. The control is chosen basis on the significant parameter used as the bifurcation parameter (Adi \& Thobirin, 2020). We introduce a control function $u(t)$, which represents the effort of tuberculosis prevention, such as vaccination. The control model is given as follows

$$
\begin{aligned}
\frac{d M_{u}}{d t} & =\Lambda-\mu M_{u}-\frac{(1-u(t)) \beta M_{u} B}{1+\alpha B} \\
\frac{M_{i}}{d t} & =\frac{(1-u(t)) \beta M_{u} B}{1+\alpha B}-c M_{i}-\frac{k M_{i}}{1+\varepsilon M_{i}}, \\
\frac{d B}{d t} & =r c M_{i}-\gamma M_{u} B-d B
\end{aligned}
$$

where $u(t)$ represents a control strategy that cures a fraction of uninfected macrophages and reduces the rate at which macrophages leaves uninfected class towards the infected class. The control is bounded between 0 and $u_{\text {max }}$. From a medical point of view, it is realistic to assume that $u_{\max }<1$, since the vaccination is not completely effective. Let us define the set of admissible control as

$$
\Omega=\left\{u(t) \in L^{1}(0, T): 0 \leq u(t) \leq u_{\max }, \forall t \in[0, T]\right\}
$$


Then, optimal control theory is applied to determine the optimal treatment administration that will maximize the effort on tuberculosis prevention measures and the cost associated with this support. We define the set of state variables $X(t)=$ $\left(M_{u}(t), M_{i}(t), B(t)\right)$ and the objective functional as

$$
J[u]=\int_{0}^{T}\left(M_{u}(t)-M_{i}(t)-u^{2}(t)\right) d t
$$

which consider the fraction of the uninfected macrophages $\left(M_{u}\right)$ and the infected macrophages $\left(M_{i}\right)$ and the cost associated with the support of transmission measure $(u)$.

The optimal control problem is to find the control $u^{*}$ with corresponding state trajectories $X^{*}=\left(M_{u}^{*}, M_{i}^{*}, B^{*}\right)$ on the time interval [0,T], that maximizes the objective functional (3.3) subject to dynamical system constraints (3.1), that is

$$
J\left[u^{*}\right]=\max _{\Omega} J[u] .
$$

Then, to apply Pontryagin's maximal principle in Theorem 1, we define the Hamiltonian as

$$
\begin{aligned}
H\left(M_{u}, M_{i}, B, u, \lambda\right) & =M_{u}(t)-M_{i}(t)-u^{2}(t)+\lambda_{1} \frac{d M_{u}}{d t}+\lambda_{2} \frac{d M_{i}}{d t}+\lambda_{3} \frac{d B}{d t} \\
& =M_{u}(t)-M_{i}(t)-u^{2}(t)+\lambda_{1}\left(\Lambda-\mu M_{u}-\frac{(1-u(t)) \beta M_{u} B}{1+\alpha B}\right) \\
& +\lambda_{2}\left(\frac{(1-u(t)) \beta M_{u} B}{1+\alpha B}-c M_{i}-\frac{k M_{i}}{1+\varepsilon M_{i}}\right) \\
& +\lambda_{3}\left(r c M_{i}-\gamma M_{u} B-d B\right)
\end{aligned}
$$

According to Pontryagin's maximum principle, for $u^{*}$ to be an optimal solution with corresponding optimal states $X^{*}$, the following conditions must be satisfied.

$$
\begin{aligned}
& \frac{d X}{d t}=-\frac{\partial H}{\partial \lambda_{i}} \\
& \frac{\partial H}{\partial u}=0 \\
& \frac{d \lambda_{i}}{d t}=-\frac{\partial H}{\partial x}
\end{aligned}
$$

According to the optimal condition (3.6), we claim the optimal solution of system (3.1) in the following theorem.

Theorem 2. There exists an optimal control $u^{*}$ corresponding to the optimal solution $M_{u}^{*}, M_{i}^{*}, B^{*}$ that maximizes the objective function $J[u]$ over $\Omega$. Moreover, there exist adjoint variables $\lambda_{i}, i=1,2,3$, along with the transversality conditions $\lambda_{i}(T)=0$ such that

$$
\begin{aligned}
& \frac{d \lambda_{1}}{d t}=-1+\lambda_{1} \mu-\left(\lambda_{1}-\lambda_{2}\right) \frac{(1-u(t)) \beta B^{*}(t)}{1+\alpha B^{*}(t)}-\lambda_{3} \gamma B^{*}(t), \\
& \frac{d \lambda_{2}}{d t}=1+\lambda_{2}\left(c+\frac{k}{\left(1+\varepsilon M_{i}^{*}(t)\right)^{2}}-\lambda_{3} r c,\right. \\
& \frac{d \lambda_{3}}{d t}=\left(\lambda_{1}-\lambda_{2}\right) \beta \frac{\left(1-u^{*}(t)\right) M_{u}^{*}(t)}{\left(1+\alpha B^{*}(t)\right)^{2}}-\lambda_{3} d .
\end{aligned}
$$

Furthermore, associated optimal control $u^{*}$ is given by

$$
u^{*}(t)=\min \left\{u_{\max }, \max \left\{0, \frac{\beta\left(\lambda_{1}-\lambda_{2}\right) M_{u}^{*}(t) B^{*}(t)}{2\left(1+\alpha B^{*}(t)\right)}\right\}\right\}
$$

Proof. The adjoint system (3.7) is derived by taking partial derivatives of the Hamiltonian (3.5) with respect to the associated state variables so that 


$$
\frac{d \lambda_{1}}{d t}=-\frac{\partial H}{\partial M_{u}}, \quad \frac{d \lambda_{2}}{d t}=-\frac{\partial H}{\partial M_{i}}, \quad \frac{d \lambda_{3}}{d t}=-\frac{\partial H}{\partial B},
$$

together with the transversality conditions $\lambda_{i}(T)=0, i=1,2,3$. The optimal control $u^{*}$ is defined by solving $\frac{\partial H}{\partial u}=0$. This lead to the condition of optimal control: $-2 u(t)+\beta\left(\lambda_{1}-\right.$ $\left.\lambda_{2}\right) \frac{M_{u}(t) B(t)}{1+\alpha B(t)}=0$. Hence, we have

Since $u^{*}$ must belong to $\Omega$, we obtain

$$
u(t)=\frac{\beta\left(\lambda_{1}-\lambda_{2}\right) M_{u}(t) B(t)}{2(1+\alpha B(t))} .
$$

$$
u^{*}= \begin{cases}0 & \text { if } u \leq 0 \\ u & \text { if } 0 \leq u \leq 1 \\ 1 & \text { if } u \geq 1\end{cases}
$$

which can also be characterized as

$$
u^{*}(t)=\min \left\{u_{\max }, \max \left\{0, \frac{\beta\left(\lambda_{1}-\lambda_{2}\right) M_{u}^{*}(t) B^{*}(t)}{2\left(1+\alpha B^{*}(t)\right)}\right\}\right\} .
$$

This completes the proof.

\section{Numerical Simulations}

This section gives some numerical simulations by using the ode45 solver in MATLAB to demonstrate the previous section's theoretical results. We compute numerically the Theorem 2 by implementing a forward-backward fourth-order Runge-Kutta method, as described in (Campos et al., 2020). The iterative method is starting with a guess on a control variable over the time interval $[0, T]$ using a forward scheme. Then, using the transversality conditions $\lambda_{i}(t)=0$, the co-state equation (3.7) are solved by a backward scheme. Furthermore, we update the control by using the state's new values and the value from (3.8). The iterative processes are stopped if the values reach convergence.
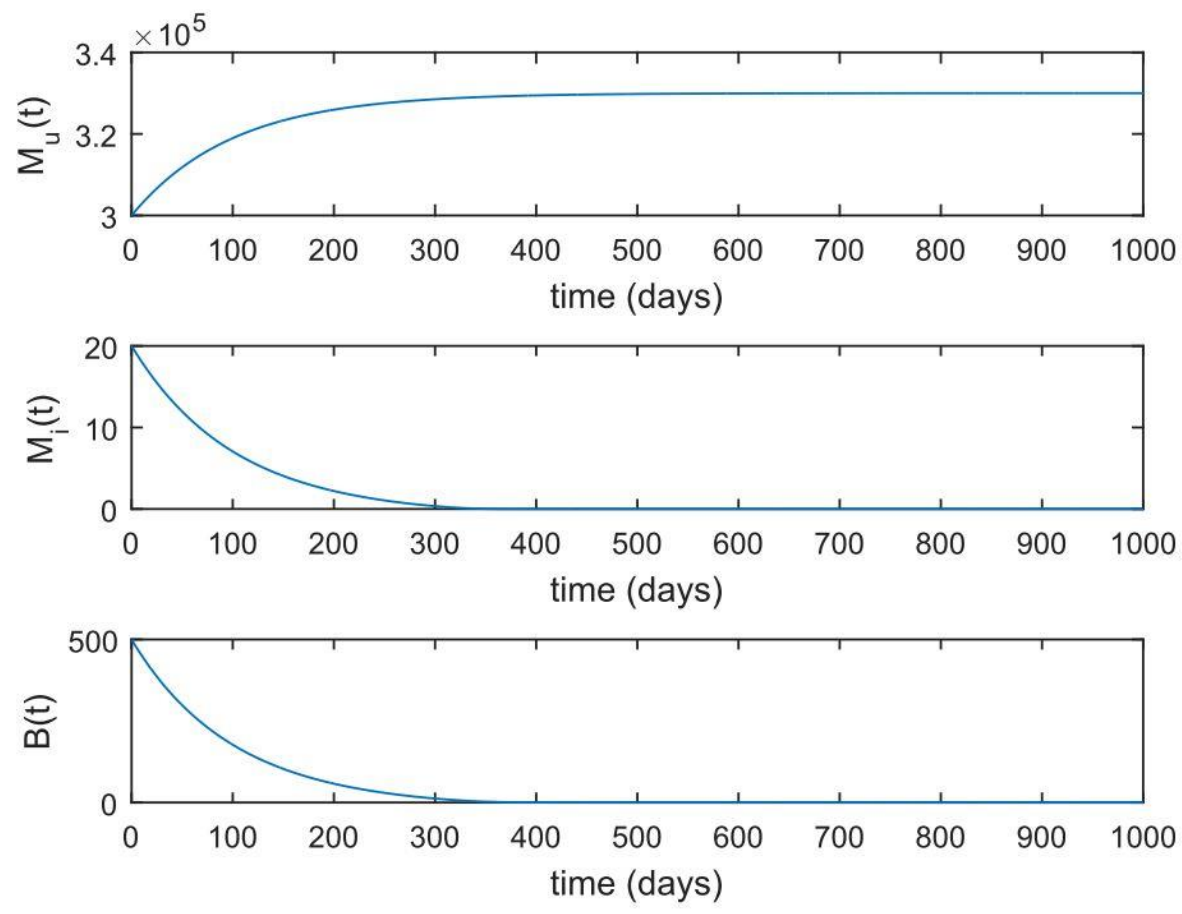

Figure 1. Solution of the System (3.4) with low transmission rates $\beta$ so that it does not require optimal control. 
For the simulation, we consider a set of parameter values obtained from the literature (Adi \& Thobirin, 2020; Zhang et al., 2020), with the unit volume in milliliters and time in days as follows

$$
\begin{gathered}
\Lambda=3300, \mu=0.01, \alpha=0.01, c=0.01, k=0.1, \varepsilon=10 \\
\quad r=100, \gamma=0.125 \times 10^{-8}, d=0.05
\end{gathered}
$$

with variation of parameter $\beta$. The initial value for the uninfected macrophage, infected macrophage, and MTb bacteria are taken as $M_{u}(0)=300000, M_{i}(0)=20$, and $B(0)=500$, respectively. Now, we consider the case of low transmission of infection rate at which the uninfected macrophages became infected and choose parameter $\beta=1.5 \times 10^{-8}$. Figure 1 shows that the infected macrophages and the MTb bacteria population are reduced, and in this case, almost no macrophages will be infected by the MTb bacteria, and the MTb bacteria become extinct. This means, in cases of very low transmission, vaccination or control is not needed, and the Mtb bacteria and infected macrophages will disappear from the bodyFigure 1 shows that infected macrophages and MTb bacteria disappear from the body in about a year (after about the 300th day). This result is very different from the model without control in (Adi \& Thobirin, 2020), where the value of the parameter $\beta=$ $2.28 \times 10^{-8}$ which is smaller than $1.5 \times 10^{-8}$ will results in the stable disease equilibrium point.

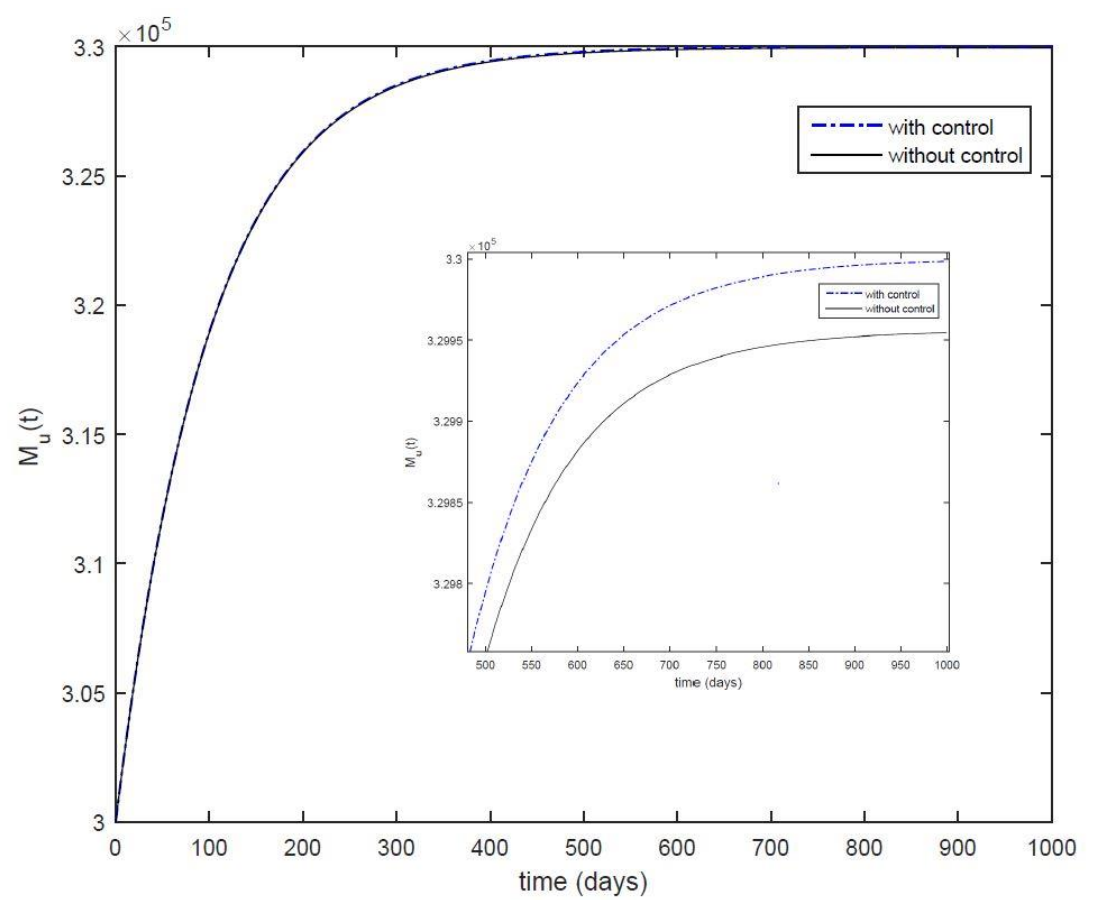

Figure 2. Results from optimal control with a set of parameter (4.1) and $\beta=1.5 \times 10^{-7}$ of uninfected macrophages, in dashed, compared with that of no control (solid). 

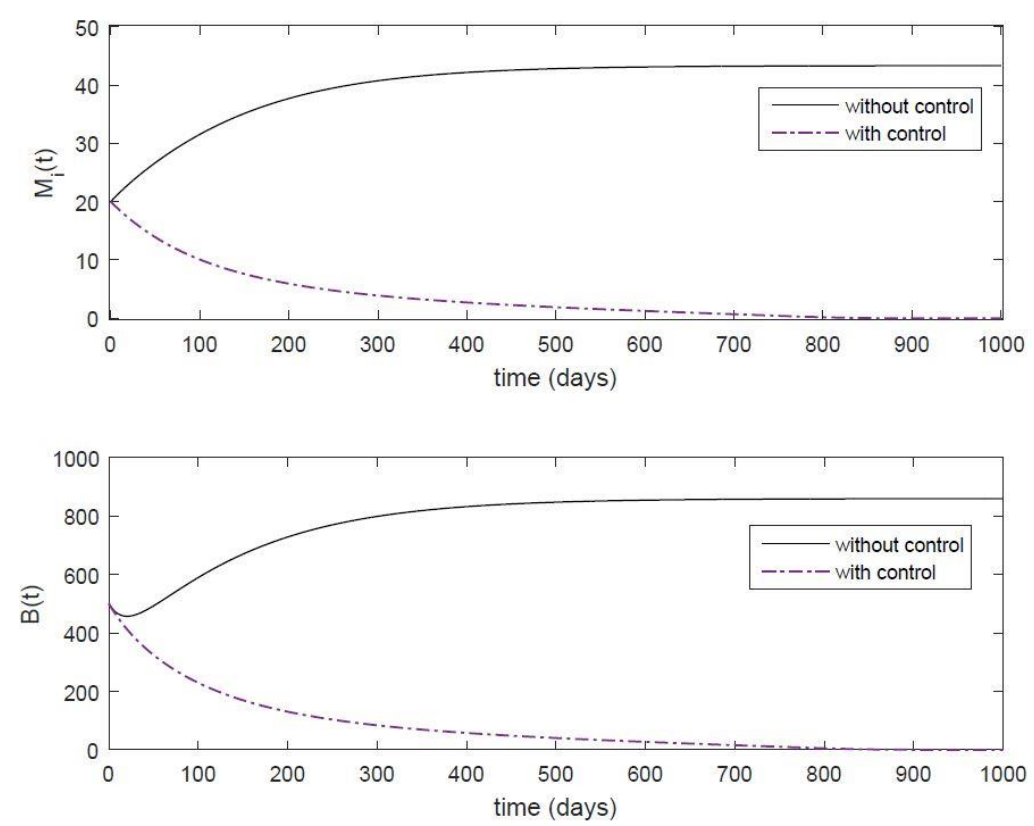

Figure 3. Results from optimal control with a set of parameter (4.1) and $\beta=1.5 \times 10^{-7}$ of infected macrophages and MTb bacteria, in dashed, compared with that of no control (solid).

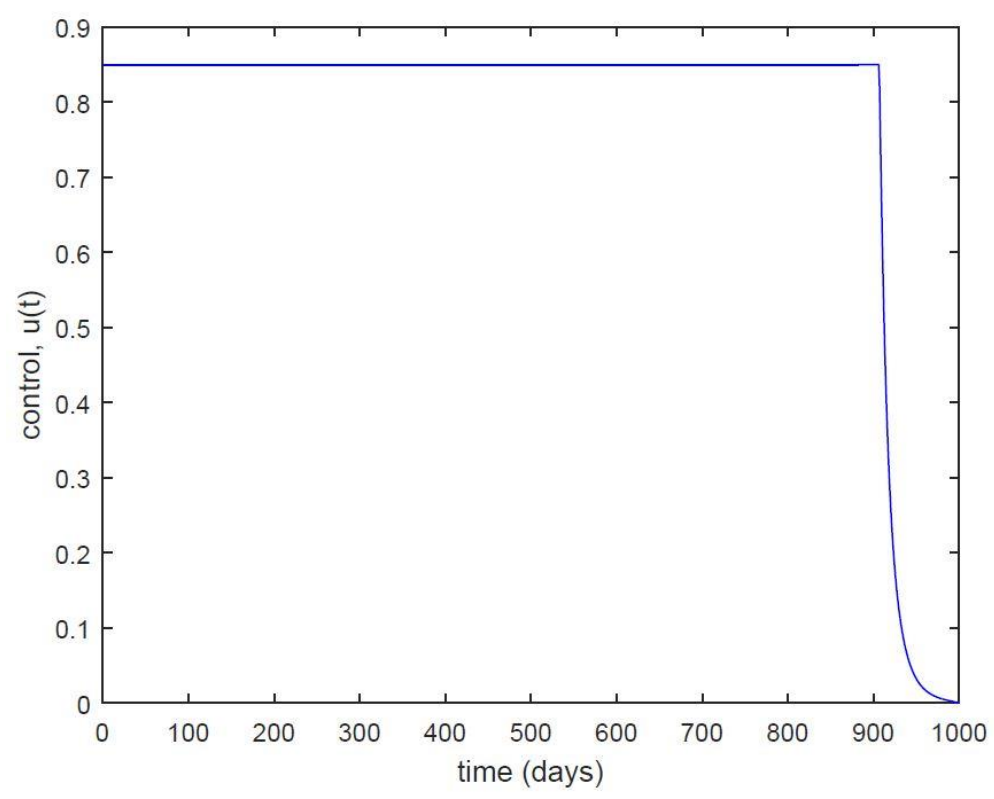

Figure 4. Optimal control $u^{*}$ for the optimal control problem (3.4) subject to the initial condition $M_{u}(0)=300000, M_{i}(0)=20$, and $B(0)=500$ and the admissible control $\Omega$.

Now, we vary $\beta$ with a much higher transmission rate, which is about ten times as much, and keep all other parameter values as in (4.1). Since vaccination is not completely effective, in this simulation, we set $u_{\max }=0.85$. Figure 2-3 show comparison trajectories for uninfected macrophages, infected macrophages, and MTb bacteria with and without control. Meanwhile, Figure 4 shows control variate over time. With the control, the number of uninfected macrophage populations is higher than without control. The increase in the number of uninfected macrophages is proportional to the decrease in the number of infected macrophages. As shown in Figure 3, with the initial condition of infected macrophages 20, without control, this number will increase to its maximum level in less than two years. Likewise, MTb bacteria's population continued to grow until it was 
at a constant level for less than two years. Meanwhile, if given control, both population infected macrophages and MTb Bacteria will decrease and disappear from the body in about two years. Appropriate control is shown in Figure 4, and a control policy is obtained; in this case, the complete vaccination is given for approximately 900 days (30 months) then decreases within 100 days later.

\section{CONCLUSION AND SUGGESTIONS}

This paper has studied an optimal control problem for a within-host tuberculosis model describing the interaction between Microbacterium tuberculosis and macrophages. We determine the existence of optimal control analytically and characterize them using Pontryagin's maximum principle. The results suggest that control or vaccination is required if the parameter $\beta$ is large. We found that with $u_{\max }=0.85$, the complete vaccination should be given for approximately 900 days (30 months) then decreased within 100 days later. Generally speaking, we suggest that for the tuberculosis disease to be successfully eradicated, it is necessary to optimize the treatment or vaccination. In other words, there is still a need to improve medical methods and technology. From the point of view of mathematical modeling, it is still necessary to develop a more realistic in-host TB model that considers the most relevant treatment methods and uses actual data to help doctors determine the right treatment for TB patients. Our future research will learn more about this.

\section{ACKNOWLEDGEMENT}

The author would like to thank the reviewers for their valuables comments. The author would also like to thank Ahmad Dahlan University for supporting this work.

\section{REFERENCES}

Adi, Y. A., \& Thobirin, A. (2020). Backward bifurcation in a within-host tuberculosis model. Advances in Mathematics: Scientific Journal. https://doi.org/10.37418/amsj.9.9.76

Agusto, F. B., \& Adekunle, A. I. (2014). Optimal control of a two-strain tuberculosis-HIV/AIDS co-infection model. BioSystems. https://doi.org/10.1016/j.biosystems.2014.03.006

Baba, I. A., Abdulkadir, R. A., \& Esmaili, P. (2020). Analysis of tuberculosis model with saturated incidence rate and optimal control. Physica A: Statistical Mechanics and Its Applications. https://doi.org/10.1016/j.physa.2019.123237

Becker, R. A., Sierstad, A., Sydsæter, K., \& Sydsaeter, K. (1989). Optimal Control Theory with Economic Applications. The Scandinavian Journal of Economics. https://doi.org/10.2307/3440172

Blaser, N., Zahnd, C., Hermans, S., Salazar-Vizcaya, L., Estill, J., Morrow, C., Egger, M., Keiser, O., \& Wood, R. (2016). Tuberculosis in Cape Town: An age-structured transmission model. Epidemics. https://doi.org/10.1016/j.epidem.2015.10.001

Bowong, S. (2010). Optimal control of the transmission dynamics of tuberculosis. Nonlinear Dynamics. https://doi.org/10.1007/s11071-010-9683-9

Brooks-Pollock, E., Roberts, G. O., \& Keeling, M. J. (2014). A dynamic model of bovine tuberculosis spread and control in Great Britain. Nature. https://doi.org/10.1038/nature13529

Byrne, A. L., Marais, B. J., Mitnick, C. D., Lecca, L., \& Marks, G. B. (2015). Tuberculosis and chronic respiratory disease: A systematic review. In International Journal of Infectious Diseases. https://doi.org/10.1016/j.ijid.2014.12.016

Campos, C., Silva, C. J., \& Torres, D. F. M. (2020). Numerical optimal control of HIV transmission in OCTAVe/MATLAB. Mathematical and Computational Applications. https://doi.org/10.3390/mca25010001

Chambers, M. L., Pontryagin, L. S., Boltyanskii, V. G., Gamkrelidze, R. V., Mishchenko, E. F., \& 
Brown, D. E. (1965). The Mathematical Theory of Optimal Processes. OR. https://doi.org/10.2307/3006724

Choi, S., Jung, E., \& Lee, S. M. (2015). Optimal intervention strategy for prevention tuberculosis using a smoking-tuberculosis model. Journal of Theoretical Biology. https://doi.org/10.1016/j.jtbi.2015.05.022

Emvudu, Y., Demasse, R., \& Djeudeu, D. (2011). Optimal control of the lost to follow up in a tuberculosis model. Computational and Mathematical Methods in Medicine. https://doi.org/10.1155/2011/398476

Fatmawati, Dyah Purwati, U., Riyudha, F., \& Tasman, H. (2020). Optimal control of a discrete age-structured model for tuberculosis transmission. Heliyon. https://doi.org/10.1016/j.heliyon.2019.e03030

Gao, D. peng, \& Huang, N. jing. (2018). Optimal control analysis of a tuberculosis model. Applied Mathematical Modelling. https://doi.org/10.1016/j.apm.2017.12.027

Kaufman, H. (1964). The Mathematical Theory of Optimal Processes, by L. S. Pontryagin, V. G. Boltyanskii, R. V. Gamkrelidze, and E. F. Mishchenko. Authorized Translation from the Russian. Translator: K. N. Trirogoff, Editor: L.. W. Neustadt. Interscience Publishers (division. Canadian Mathematical Bulletin. https://doi.org/10.1017/s0008439500032112

Kim, S., de los Reyes, A. A., \& Jung, E. (2018). Mathematical model and intervention strategies for mitigating tuberculosis in the Philippines. Journal of Theoretical Biology. https://doi.org/10.1016/j.jtbi.2018.01.026

Kuddus, M. A., Meeha, M. T., Whit, L. J., McBryd, E. S., \& Adekunl, A. I. (2020). Modeling drugresistant tuberculosis amplification rates and intervention strategies in Bangladesh. PLoS ONE. https://doi.org/10.1371/journal.pone.0236112

Kyu, H. H., Maddison, E. R., Henry, N. J., Ledesma, J. R., Wiens, K. E., Reiner, R., Biehl, M. H., Shields, C., Osgood-Zimmerman, A., Ross, J. M., Carter, A., Frank, T. D., Wang, H., Srinivasan, V., Abebe, Z., Agarwal, S. K., Alahdab, F., Alene, K. A., Ali, B. A., ... Murray, C. J. L. (2018). Global, regional, and national burden of tuberculosis, 1990-2016: Results from the Global Burden of Diseases, Injuries, and Risk Factors 2016 Study. The Lancet Infectious Diseases. https://doi.org/10.1016/S1473-3099(18)30625-X

Moualeu, D. P., Weiser, M., Ehrig, R., \& Deuflhard, P. (2015). Optimal control for a tuberculosis model with undetected cases in Cameroon. Communications in Nonlinear Science and Numerical Simulation. https://doi.org/10.1016/j.cnsns.2014.06.037

Seierstad, A., \& Sydsaeter, K. (1977). Sufficient Conditions in Optimal Control Theory. International Economic Review. https://doi.org/10.2307/2525753

Zhang, W. (2020). Analysis of an in-host tuberculosis model for disease control. Applied Mathematics Letters. https://doi.org/10.1016/j.aml.2019.07.014

Zhang, W., Frascoli, F., \& Heffernan, J. (2020). Analysis of solutions and disease progressions for a within-host Tuberculosis model. Mathematics in Applied Sciences and Engineering. https://doi.org/10.5206/mase/10221 